\title{
Racial Disparities in Oropharyngeal Cancer Stage at Diagnosis
}

\author{
UCHECHUKWU C. MEGWALU and YIFEI MA \\ Department of Otolaryngology-Head and Neck Surgery, Stanford \\ University School of Medicine, Stanford, CA, U.S.A.
}

\begin{abstract}
Aim: To evaluate the impact of race on disease stage at diagnosis in patients with oropharyngeal cancer. Patients and Methods: The cohort included 18,791 adult patients diagnosed with oropharyngeal squamous cell carcinoma between 2004 and 2012, from the Surveillance, Epidemiology, and End Results 18 Database. Results: After adjusting for age, sex, marital status, tumor site, and year of diagnosis, black race was associated with increased risk of presenting with Stage III or IV disease (OR 1.24, p=0.016), T3 or T4 tumors (OR 2.16, <0.001), distant metastasis (OR $2, p<0.001)$, and unresectable tumors (OR 1.65, $p<0.001)$. Race was not associated with risk of presenting with nodal metastasis diagnosis (OR 0.93, $p=0.241$ ). Conclusion: Black race is associated with increased risk of advanced disease presentation in oropharyngeal cancer.
\end{abstract}

Oropharyngeal cancer accounts for $10-12 \%$ of all upper aerodigestive tract cancers (1). The incidence of oropharyngeal cancer is rising (2); approximately 14,000 cases were diagnosed in the United States in 2013, accounting for 2,400 deaths (3). The majority of oropharyngeal cancers present at advanced stages (4). In addition to tumor-specific and treatment factors, non-clinical factors, such as race, gender, and socioeconomic status, may impact patient outcomes in oropharyngeal cancer.

Several studies have revealed racial disparities in head and neck cancer outcomes. Black patients are more likely to be diagnosed with more advanced disease, are less likely to receive adequate treatment, and have worse survival outcomes than other racial groups (5-8). However, African Americans also have lower incidence of oropharyngeal

Correspondence to: Uchechukwu C. Megwalu, MD, MPH, Department of Otolaryngology - Head and Neck Surgery, Stanford University School of Medicine, 801 Welch Road, Stanford, CA 94305, U.S.A. Tel: +1 6507236880, Fax: +1 6507258502, e-mail: megwaluu@yahoo.com

Key Words: Oropharynx cancer, head and neck cancer, health status disparities, minority health, SEER Program. cancer, compared to whites (9). To our knowledge, no previous study has investigated racial disparities in stage of disease at diagnosis in oropharyngeal cancer patients.

The goal of the present study was to evaluate the impact of race on disease stage at diagnosis in patients with oropharyngeal cancer, using a large population-based cancer database. We hypothesized that black patients are more likely to present with advanced stage disease and potentially incurable disease.

\section{Patients and Methods}

Data were extracted from the Surveillance, Epidemiology, and End Results (SEER) 18 Database of the National Cancer Institute, which includes data obtained from 18 population-based cancer registries in the United States. The study cohort included patients diagnosed with oropharyngeal squamous cell carcinoma between 2004 and 2012. The following International Classification of Diseases for Oncology codes were included: C01.9 (Base of tongue, NOS), C02.4 (Lingual tonsil), C05.1 (Soft palate, NOS), C05.2 (Uvula), C09.0 (Tonsillar fossa), C09.1 (Tonsillar pillar), C09.8 (Overlapping lesion of tonsil), C09.9 (Tonsil, NOS), C10.0 (Vallecula), C10.2 (Lateral wall of oropharynx), C10.3 (Posterior wall of oropharynx), (C10.8-Overlapping lesion of oropharynx), and C10.9 (Oropharynx, NOS). Exclusion criteria included multiple primary tumors, cases in which the race was recorded as "Unknown," and cases in which the mode of therapy was unknown.

Race was recorded in the SEER database as "White"; "Black"; "Other: American Indian, Alaska Native, Asian/Pacific Islander"; or "Unknown". For analysis, race was dichotomized to "Black," and "White/Other." Marital status was grouped as "married" (including common law) or "single" (single-never married, divorced, widowed). Tumor site was grouped as "base of tongue," "soft palate," "tonsil," "pharyngeal wall," and "Other." Unresectable tumor was defined as T4b or N3 tumor. All cases were coded using AJCC staging 6th edition (10).

The SEER computer software (SEER*Stat version 8.3.2; National Cancer Institute, Bethesda, Maryland, USA; Information Management Services, Inc., Calverton, Maryland, USA) was used to extract data from the SEER database. The statistical analysis was performed using SAS system, version 9.4 (SAS Institute, Inc., Cary, NC, USA). Candidate covariates for the analysis included demographic characteristics (race, age and gender), marital status, and site (tongue base, soft palate, tonsil, pharyngeal wall, and other). The outcome variables included AJCC stage group, T stage, 
presence of nodal metastasis, presence of distant metastasis, and presence of unresectable tumor. Pearson Chi Square test was used for univariable analysis comparing outcomes by race. Adjusted logistic regression model was used for multivariable analysis. Race, age, gender, marital status, site and year of diagnosis were entered a priori into the model. Missing values were handled by using logistic regression to perform multiple imputation for monotone missing binary data. Depending on the percentage of missing values, ten to 25 repetitions were used to ensure relative efficiency of the models. An estimate was considered statistically significant at $\alpha=0.05$. This study was exempt from review by the Stanford University School of Medicine Institutional Review Board because it was conducted using de-identified public data.

\section{Results}

Univariable analysis. From 2004 to 2012, the SEER database identified 18,791 patients meeting the inclusion criteria. The patients' characteristics are displayed in Table I. Black patients had higher risk of presenting with advanced T stage (T3 and T4) tumors (OR 2.55, 95\% CI 2.29 to 2.84, $p<0.0001$ ), distant metastasis (OR 2.25, 95\% CI 1.88 to $2.70, p<0.0001$ ), and unresectable tumors (OR 2, 95\% CI 1.76 to $2.29, p<0.0001)$ than other racial groups. Black patients had decreased risk of nodal metastasis (OR 0.88 , $95 \%$ CI 0.79 to $0.99, p=0.03)$. Race had no statistically significant impact on risk of Stages III or IV disease (OR $1.17,95 \%$ CI 0.99 to $1.37, p=0.07)$.

Multivariable analysis. The results of the multivariable analysis of factors predictive of advanced disease stage are shown in Table II. Black race was associated with increased risk of presenting with Stage III or IV disease, after adjusting for age, sex, marital status, tumor site, and year of diagnosis (OR 1.24, $p=0.016$ ). Single marital status (OR 1.24, $p<0.001)$ was also associated with increased risk of presenting with Stage III or IV disease, while increasing age (OR 0.92 per 5 -year increment, $p<0.001$ ) and female sex (OR $0.58, p<0.001$ ) were associated with decreased risk of presenting with Stage III or IV disease. Black race was associated with increased risk of presenting with T3 or T4 tumors, after adjusting for age, sex, marital status, tumor site, and year of diagnosis (OR 2.16, $p<0.001$ ). Single marital status (OR 1.75, $p<0.001)$ and increasing age (OR 1.04 per 5 -year increment, $p<0.001)$ were also associated with increased risk of presenting with $\mathrm{T} 3$ or T4 tumors, while female sex (OR 0.86, $p=0.002$ ) was associated with decreased risk of presenting with $\mathrm{T} 3$ or T4 tumors. There was no statistically significant association between black race and risk of presenting with nodal metastasis, after adjusting for age, sex, marital status, tumor site, and year of diagnosis (OR 0.93, $p=0.241$ ). Increasing age (OR 0.89 per 5 -year increment, $p<0.001$ ) and female sex (OR 0.66, $p<0.001)$ were associated with decreased risk of presenting with nodal metastasis.
Table I. Patients' characteristics $(n=18791)$.

\begin{tabular}{lc}
\hline Variable & \\
\hline Female & $3341(17.8 \%)$ \\
Mean Age (SD) & $59.1(10.6)$ \\
Race & \\
White & $16181(86.1 \%)$ \\
Black & $2009(10.7 \%)$ \\
Other & $601(3.2 \%)$ \\
Marital Status & \\
Married & $10049(53.5 \%)$ \\
Single & $7779(41.4 \%)$ \\
Unknown & $963(5.1 \%)$ \\
Site & \\
Tongue base & $7707(41 \%)$ \\
Soft palate & $810(4.3 \%)$ \\
Tonsil & $8881(47.3 \%)$ \\
Pharyngeal wall & $281(1.5 \%)$ \\
Other & $1112(5.9 \%)$ \\
\hline
\end{tabular}

The results of the multivariable analysis of factors predictive of untreatable disease are shown in Table III. Black race was associated with increased risk of presenting with distant metastasis, after adjusting for age, sex, marital status, tumor site, and year of diagnosis (OR 2, $p<0.001$ ). Single marital status (OR 1.67, $p<0.001)$ and increasing age (OR 1.1 per 5year increment, $p<0.001)$ were also associated with increased risk of presenting with distant metastasis, while female sex (OR 0.78, $p=0.017$ ) was associated with decreased risk of presenting with distant metastasis. Black race was associated with increased risk of presenting with unresectable tumor, after adjusting for age, sex, marital status, tumor site, and year of diagnosis (OR 1.65, $p<0.001)$. Single marital status (OR 1.64, $p<0.001)$ was also associated with increased risk of presenting with unresectable tumor, while increasing age (OR 0.97 per 5-year increment, $p=0.02$ ) and female sex (OR 0.78, $p=0.001$ ) were associated with decreased risk of presenting with unresectable tumor.

\section{Discussion}

The results of the present study show that race is associated with advanced disease presentation in oropharyngeal cancer. Black race was associated with increased risk of presenting with Stage III or IV disease, T3 or T4 tumors, distant metastasis, and unresectable tumor. Although univariable analysis suggested that black race was associated with decreased risk of presenting with nodal metastasis, this was not significant on multivariable analysis.

Only few studies have examined racial disparities in oropharyngeal cancer. Weatherspoon et al. examined oral cavity and oropharyngeal cancer incidence trends and 
Table II. Factors predictive of advanced disease stage.

\begin{tabular}{|c|c|c|c|c|c|}
\hline Outcome & Variable & Coefficient & Wald $\chi^{2}$ & Odds Ratio (95\% CI) & $p$-Value \\
\hline \multirow[t]{10}{*}{ Stage III or IV Disease } & Black race & 0.19 & 4.85 & $1.24(1.04,1.47)$ & 0.016 \\
\hline & Age (5-yr increments) & -0.08 & 50.07 & $0.92(0.90,0.94)$ & $<0.001$ \\
\hline & Female sex & -0.54 & 83.26 & $0.58(0.52,0.65)$ & $<0.001$ \\
\hline & Single marital status & 0.21 & 14.82 & $1.24(1.11,1.38)$ & $<0.001$ \\
\hline & Unknown marital status & -0.04 & 0.14 & $0.96(0.76,1.22)$ & 0.763 \\
\hline & Site: soft palate & -1.67 & 335.44 & $0.19(0.15,0.23)$ & $<0.001$ \\
\hline & Site: tonsil & -0.11 & 4.17 & $0.89(0.80,0.99)$ & 0.037 \\
\hline & Site: pharyngeal wall & -0.27 & 1.98 & $0.77(0.54,1.11)$ & 0.164 \\
\hline & Site: other & 0.40 & 8.81 & $1.47(1.12,1.95)$ & 0.007 \\
\hline & Year of diagnosis & 0.02 & 5.57 & $1.02(1.00,1.04)$ & 0.014 \\
\hline \multirow[t]{10}{*}{$\mathrm{T} 3$ or T4 Tumor } & Black race & 0.77 & 185.98 & $2.16(1.93,2.41)$ & $<0.001$ \\
\hline & Age (5-yr increments) & 0.04 & 17.52 & $1.04(1.02,1.06)$ & $<0.001$ \\
\hline & Female sex & -0.15 & 10.03 & $0.86 \quad 0.79,0.95)$ & 0.002 \\
\hline & Single marital status & 0.56 & 226.90 & $1.75(1.64,1.88)$ & $<0.001$ \\
\hline & Unknown marital status & 0.29 & 10.62 & $1.32(1.10,1.59)$ & 0.003 \\
\hline & Site: soft palate & -0.14 & 2.58 & $0.87(0.72,1.05)$ & 0.142 \\
\hline & Site: tonsil & -0.06 & 2.65 & $0.94(0.87,1.02)$ & 0.121 \\
\hline & Site: pharyngeal wall & 0.92 & 43.94 & $2.50(1.91,3.26)$ & $<0.001$ \\
\hline & Site: other & 1.35 & 264.56 & $3.80(3.25,4.46)$ & $<0.001$ \\
\hline & Year of diagnosis & -0.04 & 29.71 & $0.96(0.95,0.98)$ & $<0.001$ \\
\hline \multirow[t]{10}{*}{ Nodal Metastasis } & Black race & -0.06 & 0.87 & $0.93(0.82,1.05)$ & 0.241 \\
\hline & Age (5-yr increments) & -0.12 & 166.76 & $0.89(0.87,0.90)$ & $<0.001$ \\
\hline & Female sex & -0.42 & 79.53 & $0.66(0.60,0.72)$ & $<0.001$ \\
\hline & Single marital status & 0.05 & 1.56 & $1.06(0.98,1.15)$ & 0.144 \\
\hline & Unknown marital status & -0.34 & 15.75 & $0.71(0.61,0.84)$ & $<0.001$ \\
\hline & Site: soft palate & -1.71 & 450.24 & $0.18(0.15,0.21)$ & $<0.001$ \\
\hline & Site: tonsil & -0.15 & 12.66 & $0.86(0.79,0.93)$ & $<0.001$ \\
\hline & Site: pharyngeal wall & -0.85 & 39.42 & $0.43(0.33,0.56)$ & $<0.001$ \\
\hline & Site: other & -0.30 & 13.02 & $0.74(0.63,0.87)$ & $<0.001$ \\
\hline & Year of diagnosis & 0.03 & 16.22 & $1.03(1.01,1.05)$ & $<0.001$ \\
\hline
\end{tabular}

Table III. Factors predictive of untreatable disease.

\begin{tabular}{|c|c|c|c|c|c|}
\hline Outcome & Variable & Coefficient & Wald $\chi^{2}$ & Odds Ratio (95\% CI) & $p$-Value \\
\hline \multirow[t]{10}{*}{ Distant Metastasis } & Black race & 0.69 & 51.33 & $2.00(1.66,2.41)$ & $<0.001$ \\
\hline & Age (5-yr increments) & 0.10 & 30.69 & $1.10(1.06,1.14)$ & $<0.001$ \\
\hline & Female sex & -0.25 & 6.02 & $0.78(0.63,0.96)$ & 0.017 \\
\hline & Single marital status & 0.52 & 42.10 & $1.67(1.43,1.95)$ & $<0.001$ \\
\hline & Unknown marital status & 0.54 & 10.92 & $1.67(1.18,2.36)$ & 0.004 \\
\hline & Site: soft palate & -0.26 & 1.83 & $0.75(0.51,1.09)$ & 0.13 \\
\hline & Site: tonsil & -0.24 & 8.22 & $0.80(0.68,0.94)$ & 0.006 \\
\hline & Site: pharyngeal wall & 0.09 & 0.12 & $1.04(0.61,1.78)$ & 0.88 \\
\hline & Site: other & 0.51 & 16.25 & $1.64(1.28,2.09)$ & $<0.001$ \\
\hline & Year of diagnosis & -0.01 & 0.43 & $0.99(0.96,1.02)$ & 0.605 \\
\hline \multirow[t]{10}{*}{ Unresectable Tumor } & Black race & 0.49 & 49.50 & $1.65(1.44,1.88)$ & $<0.001$ \\
\hline & Age (5-yr increments) & -0.03 & 5.54 & $0.97(0.95,1.00)$ & 0.02 \\
\hline & Female sex & -0.25 & 13.34 & $0.78(0.68,0.90)$ & 0.001 \\
\hline & Single marital status & 0.50 & 89.32 & $1.64(1.48,1.82)$ & $<0.001$ \\
\hline & Unknown marital status & 0.17 & 1.65 & $1.17(0.91,1.50)$ & 0.226 \\
\hline & Site: soft palate & 0.13 & 0.96 & $1.13(0.88,1.45)$ & 0.338 \\
\hline & Site: tonsil & 0.38 & 44.72 & $1.47(1.31,1.65)$ & $<0.001$ \\
\hline & Site: pharyngeal wall & 0.87 & 26.56 & $2.38(1.68,3.36)$ & $<0.001$ \\
\hline & Site: other & 1.33 & 219.78 & $3.75(3.15,4.48)$ & $<0.001$ \\
\hline & Year of diagnosis & -0.05 & 30.14 & $0.95(0.93,0.96)$ & $<0.001$ \\
\hline
\end{tabular}


disparities, using the SEER database (9). They found that whites had a higher incidence risk compared to all other race/ethnicity groups. Zandberg et al. assessed racial disparities in 1,318 patients with oral cavity, oropharyngeal, hypopharyngeal, or laryngeal tumors treated at their institution (7). Similar to our study, they found that black patients had increased risk of presenting with locally advanced tumors.

To our knowledge, this is the first study to evaluate racial disparities in disease stage at diagnosis in oropharyngeal cancer patients. Our study shows that not only do black patients have increased risk of presenting with advanced disease, they are also more likely to present with potentially incurable disease. This is significant because this higher propensity to present with incurable disease may partially account for the racial disparities observed in head and neck cancer survival, even after adjusting for AJCC stage group. There are several potential explanations for the racial differences in advanced stage at diagnosis observed in our study. African Americans are more likely to be socioeconomically disadvantaged (11). Furthermore, they are more likely to be uninsured or underinsured (12). These factors may lead to presentation with late disease due to decreased access to care.

Racial differences in HPV status may play a role. However, its impact remains unclear. The prevalence of tumor HPV status, an important prognostic factor in oropharyngeal cancer, has been shown to vary by race and socioeconomic status (SES).

Liederbach et al. examined the association between sociodemographic factors and HPV status in oropharyngeal squamous carcinoma, using the National Cancer Database (NCDB) (13). They found that white patients and patients residing in high SES communities had higher prevalence of HPV-positive tumors. Furthermore, HPV-positive tumors were more likely to present with nodal metastasis, but less likely to present with advanced $\mathrm{T}$ stage and distant metastasis. Consequently, the racial differences in stage in our study may partially reflect racial differences in HPV status. Unfortunately, the SEER database does not provide information on HPV status. Therefore, we cannot evaluate its impact as a driver for the racial disparities observed.

The present study showed that marital status was associated with risk of presenting with advanced or untreatable disease. This is consistent with findings from other studies. Inverso et al. analyzed the impact of marital status on head and neck outcomes in 51,272 patients, using the SEER database (14). They found that married patients with laryngeal and oral cancers were less likely to present with metastatic disease. Contrary to our study, they found that marital status was not associated with metastatic disease in cancers of the oropharynx, hypopharynx, and nasopharynx. This discrepancy may be due to their relatively small sample size for oropharyngeal cancer (1,357 patients). Furthermore, several studies on head and neck cancer patients have demonstrated a survival advantage for married patients $(5,6,15,16)$. The mechanism for this early presentation and survival advantage for married patients is unknown. It is possible that married patients may seek earlier medical care due to spousal pressure. In addition, married patients may have better social support and more financial resources than single patients, which can lead to improved access to care, and treatment compliance.

Our study also showed that female sex was associated with decreased risk of presenting with advanced or untreatable disease. Similarly, a study by Olsen et al., examining the impact of socioeconomic status on stage at diagnosis of head and neck cancer, also revealed decreased risk of presenting with advanced disease for female patients. The reason for this is unclear. However, gender differences in health care-seeking behavior may account for this. Several studies have shown that women are more likely than men to seek care for medical problems $(17,18)$.

The main strength of our study lies in its large sample size and diverse patient characteristics. Utilizing the SEER database allowed us to analyze a large and diverse population with outstanding quality control. The catchment areas used in the SEER database were selected for their ability to maintain a high-quality cancer reporting system and for demographic characteristics that are representative of the US population as a whole. We limited our analysis to patients diagnosed from 2004 to 2012 because the SEER database uses the 6th edition of the AJCC staging criteria only for cases diagnosed after 2003. Furthermore, limiting our analysis to this recent cohort provides information on the current state of health disparities. This study is primarily limited by the retrospective nature of the analysis. In addition, the SEER database does not provide information on patient-level socioeconomic factors, which may significantly impact disease presentation. As previously discussed, the lack of HPV status in the SEER database is also a limitation.

In conclusion, the present study shows that race is associated with advanced disease presentation in oropharyngeal cancer. Black patients have increased risk of presenting with Stage III or IV disease, T3 or T4 tumors, distant metastasis, and unresectable tumor. Further research is needed to identify the causes of advanced disease presentation in black patients, including the role of HPV status as a driver of racial disparities. This may reveal potential areas of opportunity for public health interventions aimed at improving access to care for this disadvantaged group.

\section{References}

1 D'Souza G, Kreimer AR, Viscidi R, Pawlita M, Fakhry C, Koch WM, Westra WH and Gillison ML: Case-control study of human papillomavirus and oropharyngeal cancer. N Engl J Med 356: 1944-1956, 2007. 
2 Auluck A, Hislop G, Bajdik C, Poh C, Zhang L and Rosin M: Trends in oropharyngeal and oral cavity cancer incidence of human papillomavirus (HPV)-related and HPV-unrelated sites in a multicultural population. Cancer 116: 2635-2644, 2010.

3 American Cancer Society: Cancer Facts \& Figures 2015. Atlanta, GA: American Cancer Society; 2015.

4 Surveillance, Epidemiology, and End Results (SEER) Program (www.seer.cancer.gov) SEER*Stat Database: Incidence - SEER 18 Regs Research Data + Hurricane Katrina Impacted Louisiana Cases, Nov 2011 Sub (1973-2009 varying) - Linked To County Attributes - Total U.S., 1969-2010 Counties, National Cancer Institute, DCCPS, Surveillance Research Program, Surveillance Systems Branch, released April 2012, based on the November 2011 submission. Accessed January 2, 2015.

5 Olarte LS and Megwalu UC: The impact of demographic and socioeconomic factors on major salivary gland cancer survival. Otolaryngol--Head Neck Surg Off J Am Acad Otolaryngol-Head Neck Surg 150: 991-998, 2014.

6 Saini AT, Genden EM and Megwalu UC: Sociodemographic disparities in choice of therapy and survival in advanced laryngeal cancer. Am J Otolaryngol 37: 65-69, 2016.

7 Zandberg DP, Liu S, Goloubeva O, Ord R, Strome SE, Suntharalingam M, Taylor R, Morales RE, Wolf JS, Zimrin A, Lubek JE, Schumaker LM and Cullen KJ: Oropharyngeal cancer as a driver of racial outcome disparities in squamous cell carcinoma of the head and neck: 10-year experience at the University of Maryland Greenebaum Cancer Center. Head Neck 38: 564-572, 2016.

8 Shavers VL, Harlan LC, Winn D and Davis WW: Racial/ethnic patterns of care for cancers of the oral cavity, pharynx, larynx, sinuses, and salivary glands. Cancer Metastasis Rev 22: 25-38, 2003.

9 Weatherspoon DJ, Chattopadhyay A, Boroumand S and Garcia I: Oral cavity and oropharyngeal cancer incidence trends and disparities in the United States: 2000-2010. Cancer Epidemiol 39: 497-504, 2015
10 Greene FL, Page DL, Fleming ID, Fritz AG, Balch CM, Haller DG and Morrow M: AJCC cancer staging manual. 6th ed. Springer-Verlag, 2002.

11 Williams DR, Mohammed SA, Leavell J and Collins C: Race, socioeconomic status, and health: Complexities, ongoing challenges, and research opportunities. Ann NY Acad Sci 1186: 69-101, 2010.

12 Kirby JB and Kaneda T: Unhealthy and uninsured: Exploring racial differences in health and health insurance coverage using a life table approach. Demography 47: 1035-1051, 2010.

13 Liederbach E, Kyrillos A, Wang C-H, Liu JC, Sturgis EM and Bhayani MK: The national landscape of human papillomavirusassociated oropharynx squamous cell carcinoma. Int J Cancer 140: 504-512, 2017.

14 Inverso G, Mahal BA, Aizer AA, Donoff RB, Chau NG and Haddad RI: Marital status and head and neck cancer outcomes. Cancer 121: 1273-1278, 2015.

15 Andersen ZJ, Lassen CF and Clemmensen IH: Social inequality and incidence of and survival from cancers of the mouth, pharynx and larynx in a population-based study in Denmark, 1994-2003. Eur J Cancer 44: 1950-1961, 2008.

16 de Graeff A, de Leeuw JR, Ros WJ, Hordijk GJ, Blijham GH and Winnubst JA: Sociodemographic factors and quality of life as prognostic indicators in head and neck cancer. Eur J Cancer Oxf Engl 1990 37: 332-339, 2001.

17 Thompson AE, Anisimowicz Y, Miedema B, Hogg W, Wodchis WP and Aubrey-Bassler K: The influence of gender and other patient characteristics on health care-seeking behaviour: a QUALICOPC study. BMC Fam Pract 17: 38, 2016.

18 Nabalamba A and Millar WJ: Going to the doctor. Health Rep 18: 23-35, 2007.
Received January 6, 2016

Revised January 18, 2017

Accepted January 19, 2017 